\title{
Convergent molecular defects underpin diverse neurodegenerative diseases
}

\author{
George K Tofaris, ${ }^{1}$ Noel J Buckley ${ }^{2}$
}

${ }^{1}$ Nuffield Department of Clinical Neurosciences, University of Oxford, Oxford, UK

${ }^{2}$ Department of Psychiatry, University of Oxford, Oxford, UK

\section{Correspondence to} Dr George K Tofaris, Nuffield Department of Clinical Neurosciences, John Radcliffe Hospital, University of Oxford, Oxford OX3 9DU, UK; george. tofaris@ndcn.ox.ac.uk

Received 14 November 2017 Revised 24 January 2018 Accepted 1 February 2018

Published Online First

19 February 2018

Check for updates

To cite: Tofaris GK, Buckley NJ. J Neurol Neurosurg Psychiatry 2018:89:962-969.

\section{ABSTRACT}

In our ageing population, neurodegenerative disorders carry an enormous personal, societal and economic burden. Although neurodegenerative diseases are often thought of as clinicopathological entities, increasing evidence suggests a considerable overlap in the molecular underpinnings of their pathogenesis. Such overlapping biological processes include the handling of misfolded proteins, defective organelle trafficking, RNA processing, synaptic health and neuroinflammation. Collectively but in different proportions, these biological processes in neurons or non-neuronal cells lead to regionally distinct patterns of neuronal vulnerability and progression of pathology that could explain the disease symptomology. With the advent of patientderived cellular models and novel genetic manipulation tools, we are now able to interrogate this commonality despite the cellular complexity of the brain in order to develop novel therapeutic strategies to prevent or arrest neurodegeneration. Here, we describe broadly these concepts and their relevance across neurodegenerative diseases

\section{INTRODUCTION}

Neurodegenerative diseases (NDDs) are progressive disorders with increasing prevalence in our ageing population but with no cure or lasting symptomatic therapy. These include Alzheimer's disease (AD), Parkinson's disease (PD), Huntington's disease (HD), motor neuron disease (MND), frontotemporal dementias (FTDs) and prion diseases. Over 45 million people worldwide live with dementia and up to 10 million with Parkinson's disease. In the UK alone, 850000 people are affected by dementia and 127000 have Parkinson's disease. It is estimated that the number of patients with neurodegenerative brain diseases will increase to 131.5 million by 2050 (World Alzheimer Report 2015). With an estimated $€ 160$ billion of costs of care annually in Europe alone and US $\$ 818$ billion worldwide, this translates into a huge socioeconomic burden. The need to understand the cellular and molecular mechanisms that underpin neurodegeneration has never been more urgent. Historically, these diseases have been studied in isolation as separate entities, but recently a consensus has arisen that they share many common underlying mechanisms that can be exploited to identify novel therapeutic targets. Common cellular themes include misfolded protein handling, organelle trafficking, RNA processing, synaptic health and neuroinflammation. These cellular functions share overlapping molecular aetiologies. For example, misfolding of disease-specific proteins in $\mathrm{AD}, \mathrm{PD}$, $\mathrm{HD}$ or MND disrupt inter-organelle transport or degradation and impair synaptic neurotransmission whereas genome-wide association studies identified variants in inflammation-related genes across all these diseases. Therefore, delineating the shared underlying mechanisms that may counteract these cellular perturbations has become a major goal in understanding the molecular and cellular aetiology of NDD. How such mechanisms operate in the complex environment of neuronal connections and non-neuronal cells (eg, microglia or the cerebral vasculature) to generate distinct patterns of cellular degeneration is becoming clearer from genetic and genome-wide association studies as well as better modelling and functional interrogation of gene networks. Nevertheless, it is important to recognise that each NDD also has its own distinct signature, for example, deficits in nucleoprotein transport and RNA biology is a principal feature of MND whereas lysosomal defects is a major feature of PD. In this review article, we provide an update on the broader concepts behind common themes across neurodegeneration that may be of interest to practising clinicians caring for patients with NDD.

\section{Pathology of protein misfolding}

$\mathrm{AD}$ is associated with progressive deficits in memory, cognition and behaviour while PD is primarily considered a movement disorder. However, 30\% AD sufferers develop parkinsonism and up to $80 \%$ of patients with PD for 20 years develop dementia. ${ }^{1}$ This overlap extends to the primary molecular pathology of both diseases; $\mathrm{AD}$ is characterised by extracellular deposits of $\mathrm{A} \beta$ plaques and intracellular accumulations of tau neurofibrillary tangles distributed throughout the forebrain whereas PD is characterised by accumulation of $\alpha$-synuclein in Lewy bodies (LBs) primarily within the dopaminergic neurons of the midbrain. However, LB are found in the amygdala of over half of patients diagnosed with $\mathrm{AD}$ and diffusely distributed in the cortex in dementia with LBs and patients with advanced PD, often alongside AD-type pathology. ${ }^{2}$ Similarly, neuronal inclusions of TAR-DNA binding protein 43 (TDP-43) are found in motor neurons in $\mathrm{MND}^{3}$ and cortical neurons in FTD but can also be detected alongside AD-type and LB pathology in up to a third of cases with mixed pathology. ${ }^{45}$ Further evidence of common aetiology among NDD is provided by the overlap of underlying genetic contributors. For example, the $\varepsilon 4$ allele of the $A P O E$ gene is a strong genetic risk factor for both $\mathrm{LB}$ and AD-type pathology ${ }^{67}$ and 
the microtubule-associated protein tau (MAPT) H1 haplotype is associated with both $\mathrm{AD}$ and PD. ${ }^{8}$ More recently, several lossof-function variants in the ATP-binding cassette transporter A7 $(A B C A 7)$, which is involved in the clearance of protein aggregates, have been associated with both $\mathrm{AD}$ and $\mathrm{PD} .{ }^{9}$

The strongest commonality between genetic and familial forms of the same NDD, as well as across different NDD, is the idea that misfolding of specific proteins leads to the generation of conformers with toxic gain-of-function inside or outside neurons that potentially spread to distant brain regions transynaptically or by other non-cell autonomous mechanisms. ${ }^{10}{ }^{11}$ Several genes that cause monogenic $\mathrm{AD}$ such as amyloid beta precursor protein (APP), presenilin1 (PSEN1), presenilin2 (PSEN2) as well as variants in the genome such as phosphatidylinositol bonding clathrin assembly protein (PICALM) have mapped out the pathway of $\mathrm{A} \beta$ clearance and suggested that increased production and deposition of $A \beta_{1-42}$ oligomers or fibrils in the brain of patients with sporadic $\mathrm{AD}$ may be the initiating pathogenic event. ${ }^{12}$ Similarly, tau mutations were identified in familial cases of frontotemporal dementia and tau fibrils in neurofibrillary tangles is a cardinal feature of sporadic AD. $\alpha$-Synuclein mutations or multiplications are rare causes of $\mathrm{PD}$ that share the same pathology with sporadic PD, namely the misfolding of $\alpha$-synuclein into LB. ${ }^{11}$ In motor neuron disease, insoluble forms of phosphorylated and truncated TDP-43, normally a nuclear protein, accumulate in the cytosol of motor neurons ${ }^{3}$ and bi-directionally transcribed repeat RNA in cases with hexanucleotide repeat expansion in C9orf72 lead to the accumulation of dipeptide repeat proteins in dendritic inclusions in subpopulations of neurons of the motor cortex. ${ }^{13}$ In animal models, suppression of transgenic expression of APP, mutant tau, expanded huntingtin, TDP-43 or $\alpha$-synuclein in mice was shown to arrest or even reverse pathological aggregates and phenotypic defects, supporting the notion that accumulation of these misfolded proteins is toxic whereas promoting their clearance could be beneficial. ${ }^{14-17}$ Although a toxic gain of function is a widely accepted mechanism in NDD, the precise physicochemical nature of the proteotoxic conformer has not been established but is generally considered to be a prefibrillar or fibrillar oligomer rather than the end-stage compact aggregates.

These pathological changes are thought to predate the clinical presentation by several years. For example, studies of a familial AD cohort (the Dominantly Inherited Alzheimer Network (DIAN)) suggest that $A \beta_{1-42}$ levels in the cerebrospinal fluid (CSF) begin to decline as early as 25 years before the onset of symptoms. ${ }^{18}$ This is followed by the appearance of fibrillar amyloid deposits in the brain detected by PiB-PET, increased levels of tau in CSF and progressive brain atrophy roughly 15 years before clinical presentation. ${ }^{18}$ Cerebral hypometabolism and subtle episodic verbal memory impairment seem to begin about 10 years before overt dementia. ${ }^{18}$ This time course may be generally similar to that of sporadic $\mathrm{AD}$, based on cross-sectional studies ${ }^{19}$ suggesting that detectable biochemical and histopathological abnormalities occur at least two decades before clinical symptoms. A similarly protracted preclinical course has also been suggested in PD, HD and MND.

The non-cell autonomous effects of fibrillar assemblies of $\mathrm{A} \beta_{1-42}$, tau and $\alpha$-synuclein are reminiscent of 'prion-like' phenomena. The strongest evidence that this may occur in human brain comes from the identification of LB pathology in embryonic neural grafts $12-16$ years after transplantation into the brains of people with PD. ${ }^{20} 21$ Direct inoculation of human brain extract or human brain-extracted fibrils from patients with LB pathology or multi-system atrophy led to progressive $\alpha$-synuclein aggregation and neurodegeneration in connected areas of the brain of animal models including non-human primates. ${ }^{22-24}$ The propagation of $\alpha$-synuclein in mouse brain has also been demonstrated with recombinant fibrils, but cell loss in this model was not always seen. ${ }^{22} 2526$ Similarly, tau pathology without neurodegeneration was observed in wild-type or transgenic mice expressing human tau after seeding with fibrils extracted from human brain or mouse brain expressing the pathogenic P301S tau mutation. ${ }^{27}{ }^{28}$ Intracerebral infusion of dilute A $\beta$-rich brain extract from patients with $\mathrm{AD}$ or from aged APP-transgenic mice also stimulated the premature formation of plaques and amyloid angiopathy in these models. ${ }^{29}$ Induction of $\alpha$-synuclein and tau aggregation in the brain has been demonstrated following intraperitoneal or intravascular injection of fibrils. ${ }^{27}{ }^{28} 30$ Propagation of misfolded SOD1 and TDP-43 was also reported in animal models of MND. ${ }^{3132}$

Progress in identifying pathogenic proteins and their mode of propagation opened up the possibility for targeted therapies aimed at preventing their spread or promoting the clearance of their misfolded conformers. However, the most widely tested therapeutic approach to date, the use of active or passive immunotherapy, has not shown adequate clinical efficacy even though there was evidence in some trials that such approaches promote the clearance of $\mathrm{A} \beta_{1-42}{ }^{12} \mathrm{~A}$ similarly poor response was detected in a larger trial that examined the effect of $A \beta_{1-42}$ immunotherapy in a group of patients with mild AD-type dementia (EXPEDITION3). Whether such therapies need to be given at the presymptomatic phase of the disease to be effective is currently being investigated in around 300 presymptomatic members of a large Colombian pedigree with the PSEN1E280 $\rightarrow \mathrm{A} 280$ missense mutation and a smaller number of presymptomatic American participants from the DIAN cohort who carry other presenilin mutations. In contrast to the disappointing results in clinical endpoints, immunotherapies in preclinical models effectively reduced the burden of extracellular amyloid plaques and this effect has also been observed in human cases that reached post mortem. ${ }^{33}{ }^{34}$ The reason for this discrepancy is currently unclear but suggests that the complex cellular states of the human disease brain beyond the culprit protein need to be considered.

\section{Proteostasis}

One limitation of targeted therapies against misfolded proteins, as exemplified by immunotherapies, is the assumption that during 'degenerative' cellular states in the sporadic forms of these diseases, the neuronal mechanisms that normally handle misfolded proteins will rapidly recover and respond adequately to further protein aggregation, which in vivo may occur over a period of hours. ${ }^{35}$ Age-related deficiencies in protein homeostasis (proteostasis) ${ }^{36}$ could contribute to the accumulation of aggregating proteins (figure 1). For example, in $\mathrm{AD}$ or $\mathrm{PD}$ brains, there is upregulation of chaperones and accumulation of proteasomal and autophagic components. ${ }^{37}$ Accumulation of misfolded proteins typically triggers the activation of chaperones, which either attempt to refold proteins or help redirect non-native conformers towards degradation by proteasomes or lysosomes. Proteasomes are large multi-subunit complexes that consist of a $19 S$ regulatory cap and a $20 S$ proteolytic core, which together assembled into the $26 \mathrm{~S}$ particle. The $19 \mathrm{~S}$ regulatory particle recognises ubiquitinated substrates, removes the ubiquitin chains and unfolds the substrate to allow entry into the $20 \mathrm{~S}$ core, where it is rapidly degraded into peptides. ${ }^{38}$ Recent work has shown that phosphorylation of the $26 \mathrm{~S}$ proteasome subunit Rpn6/PSMD11 by protein kinase A (PKA) increased the capacity of the proteasome to degrade a variety of substrates 


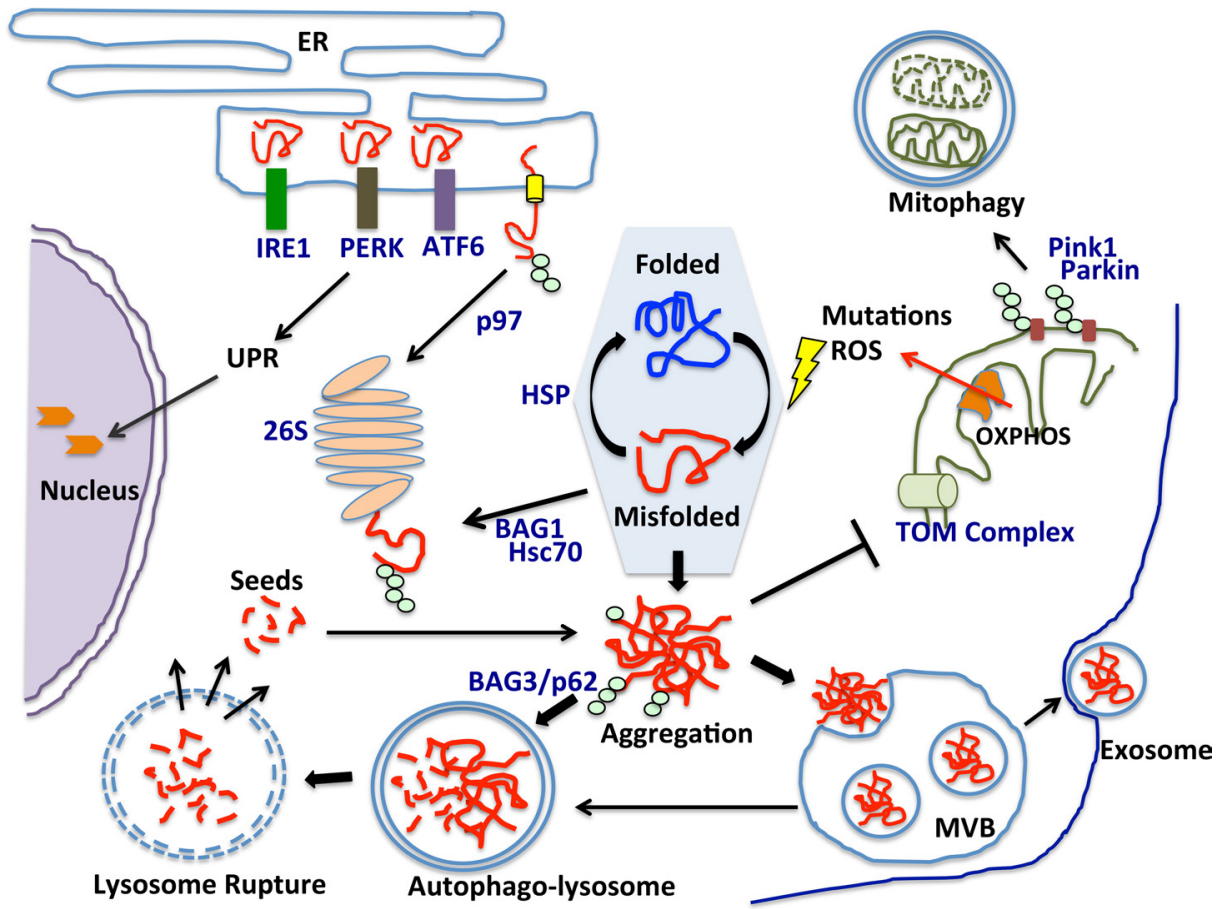

Figure 1 Proteostatic mechanisms against misfolded proteins. A central event in neurodegenerative diseases is the misfolding of diverse proteins into toxic conformers with amyloid-like structure. Misfolded proteins that cannot be refolded by chaperones (HSP) are recognised by specialised complexes such as p97 at the endoplasmic reticulum (ER) surface or BAG1/HSC70 in the cytosol and targeted for degradation by the 26S proteasome in a ubiquitin (green dots)-dependent fashion. Larger aggregates are targeted to the autophagosome, by BAG3/p62 or late endosome for degradation in the lysosome or egress via exosomes or related exocytic mechanisms. Large aggregates may escape degradation and rupture the lysosomal membrane, causing the release of proteopathic seeds that template further aggregation of soluble monomeric protein. Misfolded proteins in the ER trigger the unfolded protein response (UPR) that induces the transcription of chaperones and proteolytic pathways but if overactive can be detrimental to neurons. Misfolded proteins also impair mitochondrial function, by interfering with the import mechanisms or respiratory complexes causing the release of reactive oxygen species (ROS), which in turn promote protein misfolding. Damaged mitochondria are cleared at least in part by Pink1/Parkin-mediated mitophagy via ubiquitination of surface proteins.

including proteins involved in NDD such as mutant forms of FUS, SOD1, TDP43 and tau ${ }^{39}$ and pharmacological activation of this mechanism with rolipram translated into reduced tau-mediated cognitive decline in a transgenic mouse model. ${ }^{40}$ Although the proteasome is the primary source of protein degradation in the cell, restricted entry into the proteolytic chamber of the $20 \mathrm{~S}$ component does not permit the degradation of misfolded or large protein complexes. One way to bypass this limitation is to employ chaperone complexes such as the BAG1-HSP70 or cytosolic valosin-containing protein/p97 that retrieve misfolded proteins from aggregates and then direct them to the proteasome. Supporting the central role of such pathways in NDD is the discovery of mutations in valosin-containing protein/p97 in rare forms of ALS and FTD. ${ }^{41}$ Alternatively, larger aggregates can be directed en masse to the lysosome via autophagy. Autophagy complements the proteasome in three forms: macroautophagy, chaperone-mediated autophagy (CMA) and selective ubiquitin-dependent autophagy. Macroautophagy is the best-understood form and entails the sequestration of organelles or aggregates into a double-membrane structure known as the autophagosome. The resulting autophagosome is then transported to, and fuses with, the lysosome, delivering its cargo for degradation. In contrast, microautophagy occurs by direct engulfment of the cytosol at the lysosome membrane, and CMA occurs through HSC70-mediated delivery of proteins across the lysosomal membrane via the LAMP2A receptor. ${ }^{42}$

Selective targeting of misfolded proteins to the proteasome or lysosome is initiated by the addition of polyubiquitin chains in a three-step enzymatic process involving E1 ubiquitin-activating enzymes, E2 ubiquitin-conjugating enzymes (E2s) and E3 ubiquitin ligases (E3s). Ubiquitin immunoreactivity is detected in all pathological inclusions across NDD suggesting a role for this modification in the handling of misfolded proteins. ${ }^{43} 44$ The specificity of this system is determined by the existence of multiple E3/E2 pairs and the way they conjugate ubiquitin in chains: certain conjugates (eg, K11-linked and K48-linked chains) act as signals to the proteasome whereas others (eg, K63-linked chains) mediate trafficking to the lysosome. These ubiquitin chains are in turn recognised by adaptor complexes, which direct the ubiquitinated proteins to the relevant pathway. The relevance of ubiquitin-dependent quality control is well established in PD especially in a subgroup of familial cases that are caused by mutations in the E3 ligases Parkin or FBXO7. In addition, mutations in the ubiquitin adaptors SQSTM1/p62 or optineurin cause familial MND/FTD whereas variants in BAG3 were linked to PD. ${ }^{45} 46$

One mechanism by which ubiquitin-conjugating enzymes could mitigate toxicity in sporadic forms of NDD involves the direct ubiquitination and degradation of either monomeric or aggregated forms of pathogenic proteins as shown in reductionist systems and animal models. For example, the E3 ligase NEDD4 promotes whereas the deubiquitinase USP8 opposes the lysosomal clearance of $\alpha$-synuclein and genetic modulation of these activities reduced $\alpha$-synuclein toxicity. ${ }^{47-50}$ NEDD4 has been shown to have protective effects against TDP-43 and A $\beta^{51}$ that may be related to its broader effects on stress granule regulation 
or the activation of autophagy. ${ }^{5253}$ USP14 is a major regulator of protein turnover by the proteasome and its inhibition with small molecules accelerates the clearance of tau and TDP-43. ${ }^{54}$ Regulation of ubiquitination by USP19 has been implicated in the vesicular export of misfolded proteins at the endoplasmic reticulum (ER) that escape cytosolic degradation. ${ }^{55}$ More broadly, ubiquitin homeostasis is critical for neuronal viability. For example, partial inactivation of the deubiquitinase UCH-L1, an enzyme that maintains free ubiquitin levels, leads to neurodegeneration ${ }^{56}$ and YOD1 in association with the AAA-ATPase p97 regulates the disposal of ruptured lysosomes and their content caused by tau fibrils. 57

Impaired clearance of misfolded proteins may lead to their accumulation in the lumen of the ER. These misfolded proteins are normally recognised by the ER membrane-associated complexes and retrotranslocated to the cytoplasm for degradation by the valosin-containing protein/p97-NPL4-UFD-1 complex. In NDD, increased accumulation of misfolded proteins in the ER lumen induces ER stress and activates the unfolded protein response (UPR). This is normally a protective cellular response that aims to reduce unfolded protein load and restore protein-folding homeostasis. The UPR has three arms, which initiate signalling cascades through protein kinase RNA (PKR)like ER kinase (PERK), inositol-requiring enzyme 1 (IRE1) and activating transcription factor 6 (ATF6). Paradoxically, persistent activation of the PERK-eIF $2 \alpha$ branch of the UPR is detrimental as it causes uncompensated decline in global translation rates leading to synaptic failure and neuronal death. ${ }^{58}$ Restoring translation by targeting PERK or more selectively eIF $2 \alpha$ using repurposed drugs such as trazadone was neuroprotective and enhanced memory in animal models of prion disease and tauopathy despite the accumulation of misfolded proteins. ${ }^{59}$

\section{Inter-organelle trafficking and organelle turnover}

Defects in inter-organelle trafficking, especially ER to Golgi, endosomes to lysosomes and the turnover of lysosomes or mitochondria have emerged as critical events based on genetic and functional studies across NDD. For example, mutations in VPS35, a component of the retromer complex that functions in endosomal protein sorting cause late-onset forms of familial $\mathrm{PD}$ and $\mathrm{AD} .{ }^{60}$ Heterozygous mutations in the lysosomal enzyme glucocerebrosidase (GBA), which when biallelic cause Gaucher's disease, are the the most common risk factor for $\mathrm{PD},{ }^{61}$ at least partly by impairing the lysosomal degradation of $\alpha$-synuclein. ${ }^{62}$ Mutations in the kinase LRRK2, which is the the most common form of autosomal dominant PD, impair endosomal trafficking and autophagy and are linked pathologically to the accumulation of both LB and neurofibrillary tangles. ${ }^{60}$ Endosomal processing is also critical in the cleavage of APP and generation of $A \beta_{1-42}$ or misfolded prions. C9orf72, a commonly mutated gene in MND and FTD, regulates vesicle trafficking via an interaction with the GTPase RAB7L $1^{63}$ and progranulin, which is haploinsufficient in FTD, regulates lysosome acidification and biogenesis. ${ }^{64}$ The relevance of these pathways in sporadic NDD is reinforced by genome-wide linkage of variants in genes implicated in organelle trafficking with disease (eg, $L R R K 2, G A K$ and $R A B 7 L$ in PD and PICALM in AD).

Mitochondria provide a large proportion of ATP in the nervous system and also regulate intracellular $\mathrm{Ca}^{2+}$ homeostasis and the induction of apoptosis. Numerous studies in animal models showed that mitochondrial deficits are an early feature of diverse proteinopathies associated with NDD, ${ }^{65-68}$ especially at the synapse, where synaptic mitochondria are subjected to the high-energy requirements associated with synaptic activity. ${ }^{6869}$ The relevance of mitochondria for healthy dopaminergic neuronal function in humans is supported by observations in methyl-4-phenyl-1,2,3,6-tetrahydropyridine (MPTP) users who developed parkinsonism due to the conversion in astrocytes of MPTP to 1-methyl-4-phenylpyridinium $\left(\mathrm{MPP}^{+}\right)$, which inhibits complex I of the electron transport chain in neurons. More broadly, respiratory chain defects due to mutations in components of complex I to V (Leigh's syndrome) cause earlyonset diffuse neuronal loss pointing towards the critical role of oxidative phosphorylation for neuronal viability. In sporadic NDD, misfolded $\alpha$-synuclein, oligomeric A $\beta$ or mutant SOD1 were shown to bind to mitochondrial membranes and interfere with electron transport via interactions with complex I or IV and the TOM complex, which is responsible for the import of mitochondrial proteins. ${ }^{70}$ Wild-type and mutant forms of TDP-43 were shown to gain access to the mitochondrial matrix and bind to mRNA encoding the complex I subunits ND3 and ND6, impairing its assembly and function. ${ }^{72}$ Interactome analysis revealed that dipeptide repeat protein in neurons with repeat expansions in C9Orf72 preferentially bound to mitochondrial ribosomal proteins causing oxidative stress. ${ }^{73}$ Mutant huntingtin may lead to mitochondria dysfunction via dysregulation of key transcription factors such as the CREB binding protein. ${ }^{67} 74$ These examples serve to identify mitochondria as the recipients of cellular stressors, but their dysfunction can ultimately amplify intracellular and non-cell autonomous signals that contribute to the progression of diverse pathologies via ATP reduction and release of reactive oxygen species or apoptotic signals. Accordingly, mechanisms of mitochondrial turnover are strong genetic determinants of dopaminergic neuronal death in certain forms of PD with homozygous mutations in Pink1 or Parkin genes. In this pathway, dissipation of the mitochondrial membrane potential induces translocation of the kinase Pink1 to mitochondria and activation of Parkin through phosphorylation of ubiquitin and the ubiquitin-like domain of Parkin. This cascade initiates the degradation of mitochondrial proteins by the proteasome in a p97-dependent fashion or whole mitochondrial segments by autophagy (mitophagy). ${ }^{75}$ This pathway was shown to prevent dopaminergic degeneration in a mouse model of mitochondrial disease ${ }^{75}$ and may also serve broader protective effects as it also mitigates $A \beta$ pathology in vivo. ${ }^{76}$ It should be noted that parkin-independent mitophagy is also active in the nervous system. $^{77}$

\section{Neuroinflammation}

Although neuroinflammation has long been considered a secondary event caused by neurodegeneration, recent genomewide association studies and functional analyses in model systems suggest that microglia-related pathways are primary effectors in the pathogenesis of NDD. ${ }^{78}$ Microglia are the resident macrophages of the central nervous system. They use classic immune molecules, such as complement proteins, to signal to neurons and glia, survey their microenvironment and refine the neuronal connectivity during development, in health and disease. Their role in neurodegeneration is strengthened by the association of mutations in genes that regulate immune signalling with NDD. A prominent example is TREM2, an innate immune receptor expressed on microglia and cells of the monocyte lineage. Human genetic studies identified rare mutations in TREM2 as a risk factor for AD and potentially PD and FTD. ${ }^{79-81}$ TREM2 was shown to act as a negative regulator of inflammatory cytokine and Toll-like receptor (TLR) responses. ${ }^{82}$ Interestingly, TLR mediates the uptake of misfolded forms of $\alpha$-synuclein and $A \beta$ in microglia, which may represent an 
alternative non-cell autonomous mechanism for the progression of pathology. ${ }^{83} 84$ Previous work showed that TREM2 helps sustain a microglial response around plaques that may function to contain toxic $\mathrm{A} \beta$ conformers and protect nearby neurites. ${ }^{85} 86$ This work suggests that TREM2 signalling may be beneficial in responding to amyloid pathology, while variants leading to a loss of TREM2 function are detrimental. In contrast, lack of TREM2 in a mouse model of tauopathy was neuroprotective, reducing gliosis and neuroinflammation, which corresponded with preservation of brain volume without an effect on tau aggregation. ${ }^{87}$ If confirmed, these data would suggest that the same microglial-related signalling mechanism has contrasting actions in NDD depending on the underlying proteinopathy.

Synaptic signals also influence microglia by activating cell surface receptors and modulating ion channels. In turn, perisynaptic microglia are able to sense early disruptions in synaptic activity and potentially contribute to synaptic demise. This process has been termed synaptic pruning, during which microglia connect with synapses using their highly motile processes and engulf the neuronal terminals. ${ }^{88}$ One mechanism involved in this process is the classical complement cascade. ${ }^{89} 90$ Region-specific synapse loss and dysfunction is an early hallmark of NDD. Complement proteins are often upregulated in NDD and localise around areas of pathology, for example, in neuritic plaques, along with microglia. C1q and C3 also associate with synapses before overt plaque deposition in multiple $\mathrm{AD}$ mouse models and localise to brain regions that are vulnerable to synapse loss. ${ }^{89}$ In addition, microglia in adult mice engulf synaptic material in the presence of soluble oligomeric $A \beta$, in a complement receptor-dependent manner, and blocking the complement cascade protects the synapses from A $\beta$-induced loss. ${ }^{89}$ Interestingly, complement activation and microglia-mediated synaptic pruning are also drivers of neurodegeneration caused by progranulin deficiency in mice $^{91}$ suggesting that similar mechanisms may be at play in other NDD.

\section{RNA and neurodegeneration}

Although most NDD result primarily from defects in protein function, there is clear evidence that aberrant RNA processing is also a driver or contributing factor to the aetiology of neurodegeneration. Both coding and non-coding RNAs (including microRNAs and long non-coding RNAs (ncRNAs)) have been linked to NDD and all aspects of RNA metabolism have been implicated including splicing, stabilisation and transport (figure 2). The most prominent disease associated with defects in RNA biology is MND. Mutations in the RNA binding proteins TDP-43 ${ }^{92-94}$ and FUS ${ }^{95}$ cause familial MND, and mislocalisation of TDP-43 into cytoplasmic aggregates is a key pathogenic event in sporadic forms of the disease. ${ }^{97}$ TDP-43 plays a role in several aspects of RNA processing, most notably regulation of splicing, ${ }^{98-101}$ stability ${ }^{102}$ and transport. ${ }^{103}$ Although impaired TDP-43 and FUS-mediated regulation of the splicing of thousands of coding and non-coding RNAs contribute to neurodegeneration, the identity of specific RNAs that are causatively linked to pathology is unknown. In addition to the effects of mutant TDP-43 and FUS on aberrant splicing, cytoplasmic aggregates and

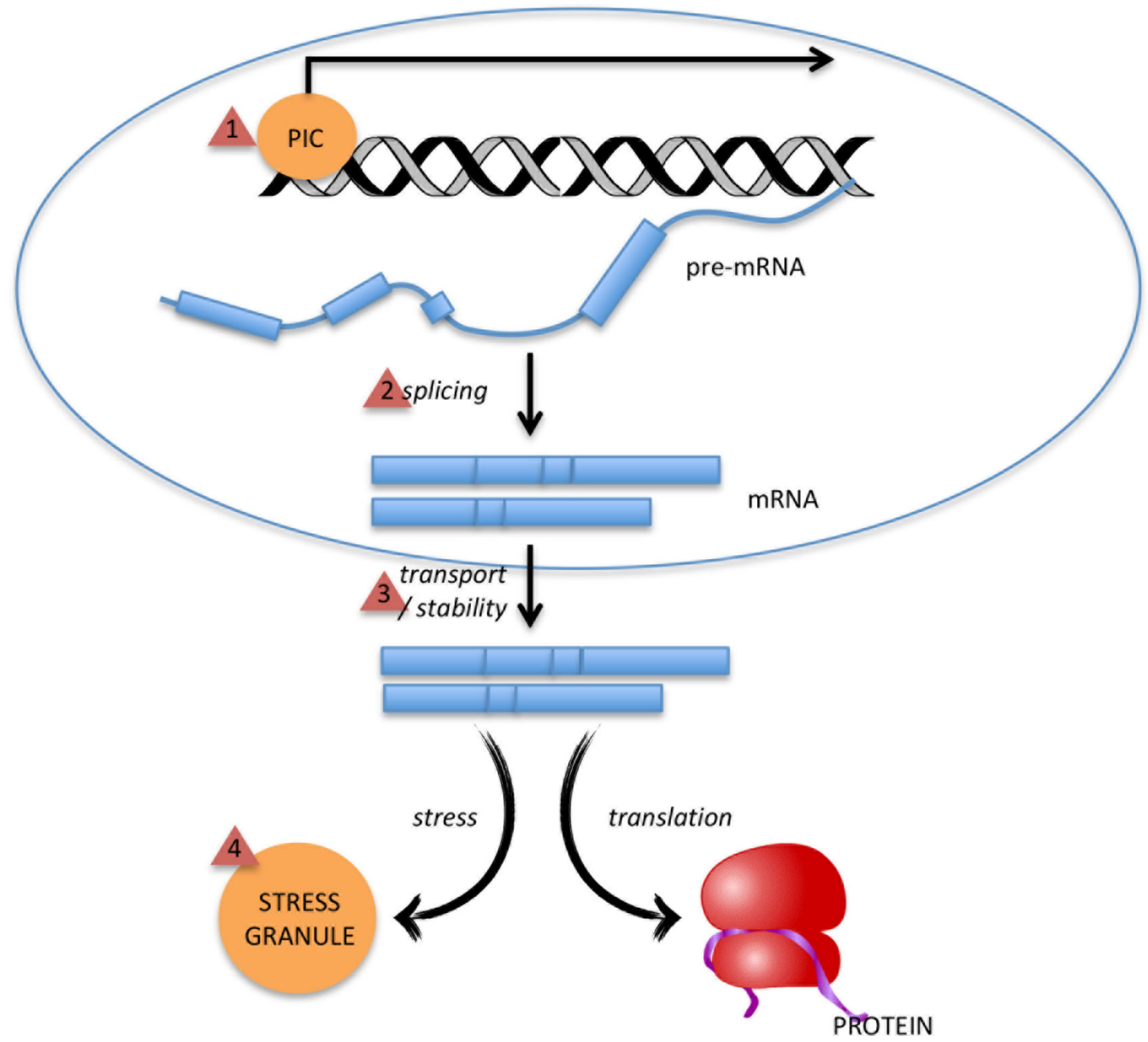

Figure 2 RNA metabolism and neurodegeneration: RNA binding proteins such as TDP43 and FUS regulate multiple levels of RNA metabolism including transcription (1), splicing (2), nucleocytoplasmic transport and stability (3) and localisation to stress granules (4). Mutations in TARDP and FUS can lead to errors in transcription, production of alternative transcripts and cellular stress, all of which lead to neuronal death. PIC, transcription preinitiation complex. 
stress granules were also shown to cause the mislocalisation of other RNA binding proteins, causing further impairment on RNA processing. ${ }^{104}$ More recently, aggregated and disease-linked mutant TDP- $43^{105}$ or RAN translated dipeptide repeat proteins as observed in C9orf72-related disease ${ }^{106}$ were shown to trigger the sequestration of nucleoporins interfering specifically with nuclear protein import and RNA export in Drosophila models, primary neurons and induced pluripotent stem cell-derived neurons from patients with TPD-43 with C9orf72-related mutations. Moreover, nuclear pore pathology was detected in brain tissue in cases of sporadic MND and those involving genetic mutations in TARDBP and C9orf72. ${ }^{105}$ These studies suggest that nucleocytoplasmic transport may be a common mechanism linking protein aggregation and RNA metabolism in sporadic and familial forms of MND/FTD.

Evidence that RNA is a significant driver of neurodegeneration also comes from studies in trinucleotide repeat disorders. ${ }^{107}$ The underlying common molecular aetiology of all such disorders is an unstable expansion of repetitive DNA, most commonly (CAG)n, which when translated gives rise to a polyglutamine (PolyQ) tract that assembles into fibrillar intranuclear or cytoplasmid inclusions. Prominent examples include the CAG expansion in the first exon of the huntingtin gene (HTT) giving rise to $\mathrm{HD}$ and the CAG expansion in ataxin 3 (ATXN3) giving rise to spinocerebellar ataxia type 3 (SCA3). The pathogenic role of RNA in SCA3 is supported by studies where interruption of the CAG expansion in ATXN3 with CAA codons (encoding glutamine) that still retain the PolyQ expansion abrogates the toxicity in fly models whereas insertion of the CAG expansion into unrelated proteins led to toxicity. ${ }^{108}$

Perhaps the complexity of the role of RNA in neurodegeneration is most directly observed when considering ncRNAs, which by definition are not translated into any protein product. For example, spinocerebellar ataxia type 8 is associated with a CAG expansion in the sense strand of ATXN8 (ataxin 8) gene and CTG expansion in the opposite strand, the latter giving rise to a CUG expansion in the AXN8OS (ataxin 8 opposite strand) antisense RNA, ${ }^{109}$ suggesting that toxicity can arise from the presence of the AXN8OS in RNA foci ${ }^{110}$ as well as expanded ATXN8 protein in nuclear inclusions. ${ }^{111}$ A similar situation exists in Huntington's like 2 (HDL2) disease caused by CTG expansions in non-coding regions of the Junctophilin-3 gene. As in SCA8, this results in a CAG expansion in an antisense transcript that is sufficient to cause the HD-like phenotype. ${ }^{112}$ Several other mechanisms have been ascribed to toxic RNA repeats including interaction with RNA binding proteins, formation of intracellular RNA foci and epigenetic regulation. ${ }^{107}$ These are not isolated contributory factors but more likely represent an interactive network linking repeat expansion in RNA and protein transcripts with toxicity.

\section{Insights from new technologies}

Until recently, the study of human neurons was largely confined to the histological characterisation of end-stage disease in postmortem brains. The advent of induced pluripotent stem cells (iPSC)-based models has enabled dynamic studies in patient-derived neurons. Unlike immortalised human cell lines, iPSCs are derived from any personal genetic background and reprogrammed from a wide range of somatic cells including skin fibroblasts and hair follicle cells making it possible to readily make patient-specific iPSCs. These, in turn, can be differentiated towards multiple neuronal and glial cell types relevant to individual NDD, associated with loss of specific neuronal populations such as cortical neurons in $\mathrm{AD}$, medium spiny GABAergic neurons in HD and dopaminergic neurons in PD. There are now numerous studies in iPSC-based models with considerable heterogeneity in research practices and extent of maturity of such iPSC-derived cells. Nevertheless, recent attempts to generate a phenogenetic on-line database (iPhemap) to catalogue disease phenotypes will greatly assist our search for and understanding of emerging common mechanisms across NDD. ${ }^{113}$ Phenotypic characterisation of iPSC-derived neurons from patients with monogenic forms of NDD has reinforced the notion that neurons exhibit similar cellular defects. For example, iPSC-derived neurons expressing mutant forms of $\alpha$-synuclein, tau, C9Orf72 or expanded huntingtin have impaired mitochondrial function causing oxidative stress. ${ }^{49}$ 114-116 ER stress was observed in iPSC lines from patients with GBA-related PD, ${ }^{117}$ $\alpha$-synuclein A53T mutation ${ }^{49}$ or triplication, ${ }^{118} \mathrm{C} \mathrm{orf}_{72} 2^{73}$ and Tau A152T. ${ }^{116}$ Whether these changes reflect the generic state of an unhealthy neuron in the dish or a common downstream effector mechanism of neuronal vulnerability to different forms of proteotoxicity remains to be seen.

Gene editing now offers the possibility of introducing or reversing disease-associated mutations or modulating the expression of modifiers. ${ }^{119}$ The most widely used CRISPR/Cas9 system ${ }^{120}{ }^{121}$ can be thought of as a dual module made up of a unique genomic address delivery system (provide by single-guide RNAs—sgRNAs) and an enzyme cargo (CRISPR associated protein 9-Cas9 nuclease). The sgRNA binds to its unique complementary DNA sequence and delivers the Cas9 to a precise locus within the genome. The Cas9 nuclease then introduces double-stranded DNA breaks, which are subsequently repaired by endogenous DNA repair mechanisms and as a consequence introduces discrete mutations into the targeted locus. More recently, numerous engineered forms of Cas9 have been introduced that use Cas9 as a delivery vehicle without nuclease activity to either enhance or suppress gene expression. These tools have greatly expanded the repertoire of genomic and epigenomic changes including (1) reversion of specific disease-associated mutations, (2) control of transcriptional activation or silencing and (3) manipulation of specific epigenetic marks. ${ }^{122}$ The combined power of iPSCs and gene editing will facilitate the dissection of causative association from correlative ones.

\section{CONCLUDING REMARKS}

Although a number of shared themes have been identified across NDD, it is likely that pathogenesis is not a linear cascade but rather a constellation of cellular pathways that define neuronal vulnerability and potentially gate the progression of pathology arising from different misfolded proteins. Nevertheless, the brain exhibits remarkable plasticity, which ensures that the myriad of its neuronal connections continue to function until a critical threshold is eventually reached. It is likely that a prolonged period of neuronal compensated dysfunction precedes cell loss. Identifying early stages on this neurodegenerative trajectory and delineating the druggable targets within such fundamental protein networks will be key to developing future therapeutic interventions. In this respect, regulators of protein misfolding, inter-organelle trafficking or neuroinflammation are emerging as potential therapeutics across NDD.

\section{Contributors GKT and NJB wrote the manuscript.}

Funding GKT is funded by a Wellcome Trust Intermediate Clinical Fellowship (097479/Z/11/Z) and the Wellcome Beit Prize fellowship (097479/Z/11/A), European Union's Horizon 2020 research and innovation programme under grant agreement no. 116060 (IMPRiND), ARUK, BMA, the Oxford Biomedical Research Centre and EPSRC (EP/M006204/1).

Disclaimer The opinion expressed and arguments employed herein do not necessarily reflect the official views of these funding bodies.

Competing interests None declared. 
Provenance and peer review Commissioned; externally peer reviewed.

(c) Article author(s) (or their employer(s) unless otherwise stated in the text of the article) 2018. All rights reserved. No commercial use is permitted unless otherwise expressly granted.

\section{REFERENCES}

1 Hely MA, Reid WG, Adena MA, et al. The Sydney multicenter study of Parkinson's disease: the inevitability of dementia at 20 years. Mov Disord 2008;23:837-44

2 Hamilton RL. Lewy bodies in Alzheimer's disease: a neuropathological review of 145 cases using alpha-synuclein immunohistochemistry. Brain Pathol 2000;10:378-84.

3 Neumann M, Sampathu DM, Kwong LK, et al. Ubiquitinated TDP-43 in frontotemporal lobar degeneration and amyotrophic lateral sclerosis. Science 2006;314:130-3

4 Uryu K, Nakashima-Yasuda H, Forman MS, et al. Concomitant TAR-DNAbinding protein 43 pathology is present in Alzheimer disease and corticobasal degeneration but not in other tauopathies. J Neuropathol Exp Neurol 2008;67:555-64

5 Nakashima-Yasuda H, Uryu K, Robinson J, et al. Co-morbidity of TDP-43 proteinopathy in Lewy body related diseases. Acta Neuropathol 2007;114:221-9

6 Lippa CF, Smith TW, Saunders AM, et al. Apolipoprotein E genotype and Lewy body disease. Neurology 1995;45:97-103.

7 Saunders AM, Schmader K, Breitner JC, et al. Apolipoprotein E epsilon 4 allele distributions in late-onset Alzheimer's disease and in other amyloid-forming diseases. Lancet 1993:342:710-1.

8 Shulman JM, De Jager PL. Evidence for a common pathway linking neurodegenerative diseases. Nat Genet 2009;41:1261-2.

9 Nuytemans K, Maldonado L, Ali A, et al. Overlap between Parkinson disease and Alzheimer disease in ABCA7 functional variants. Neurol Genet 2016;2:e44.

10 Goedert M, Eisenberg DS, Crowther RA. Propagation of Tau aggregates and neurodegeneration. Annu Rev Neurosci 2017;40:189-210.

11 Tofaris GK, Goedert M, Spillantini MG. The transcellular propagation and intracellular trafficking of $\alpha$-Synuclein. Cold Spring Harb Perspect Med 2017;7:a024380.

12 Selkoe DJ. Preventing Alzheimer's disease. Science 2012;337:1488-92.

13 Saberi S, Stauffer JE, Jiang J, et al. Sense-encoded poly-GR dipeptide repeat proteins correlate to neurodegeneration and uniquely co-localize with TDP-43 in dendrites of repeat-expanded C9orf72 amyotrophic lateral sclerosis. Acta Neuropathol 2017;122:691-16.

14 Jankowsky JL, Slunt HH, Gonzales V, et al. Persistent amyloidosis following suppression of Abeta production in a transgenic model of Alzheimer disease. PLoS Med 2005:2:e355.

15 Santacruz K, Lewis J, Spires T, et al. Tau suppression in a neurodegenerative mouse model improves memory function. Science 2005;309:476-81.

16 Lim Y, Kehm VM, Lee EB, et al. $\alpha$-Syn suppression reverses synaptic and memory defects in a mouse model of dementia with Lewy bodies. J Neurosci 2011:31:10076-87.

17 Walker AK, Spiller KJ, Ge G, et al. Functional recovery in new mouse models of ALS/FTLD after clearance of pathological cytoplasmic TDP-43. Acta Neuropatho 2015;130:643-60.

18 Bateman RJ, Xiong C, Benzinger TL, et al. Clinical and biomarker changes in dominantly inherited Alzheimer's disease. N Engl J Med 2012;367:795-804.

19 Jack CR, Knopman DS, Jagust WJ, et al. Hypothetical model of dynamic biomarkers of the Alzheimer's pathological cascade. Lancet Neurol 2010;9:119-28.

20 Li JY, Englund E, Holton JL, et al. Lewy bodies in grafted neurons in subjects with Parkinson's disease suggest host-to-graft disease propagation. Nat Med 2008:14:501-3.

21 Kordower JH, Chu Y, Hauser RA, et al. Lewy body-like pathology in long-term embryonic nigral transplants in Parkinson's disease. Nat Med 2008;14:504-6.

22 Masuda-Suzukake $M$, Nonaka T, Hosokawa $M$, et al. Prion-like spreading of pathological $\alpha$-synuclein in brain. Brain 2013;136:1128-38.

23 Recasens A, Dehay B, Bové J, et al. Lewy body extracts from Parkinson disease brains trigger $\alpha$-synuclein pathology and neurodegeneration in mice and monkeys. Ann Neurol 2014:75:351-62.

24 Prusiner SB, Woerman AL, Mordes DA, et al. Evidence for $\alpha$-synuclein prions causing multiple system atrophy in humans with parkinsonism. Proc Natl Acad Sci U SA 2015;112:E5308-E5317.

25 Luk KC, Kehm V, Carroll J, et al. Pathological $\alpha$-synuclein transmission initiates Parkinson-like neurodegeneration in nontransgenic mice. Science 2012:338:949-53.

26 Osterberg VR, Spinelli KJ, Weston LJ, et al. Progressive aggregation of alphasynuclein and selective degeneration of lewy inclusion-bearing neurons in a mouse model of parkinsonism. Cell Rep 2015;10:1252-60.

27 de Calignon A, Polydoro M, Suárez-Calvet M, et al. Propagation of tau pathology in a model of early Alzheimer's disease. Neuron 2012;73:685-97.

28 Clavaguera F, Bolmont T, Crowther RA, et al. Transmission and spreading of tauopathy in transgenic mouse brain. Nat Cell Biol 2009;11:909-13.

29 Meyer-Luehmann M, Coomaraswamy J, Bolmont T, et al. Exogenous induction of cerebral beta-amyloidogenesis is governed by agent and host. Science 2006:313:1781-4.
30 Peelaerts W, Bousset L, Van der Perren A, et al. $\alpha$-Synuclein strains cause distinct synucleinopathies after local and systemic administration. Nature 2015;522:340-4.

31 Iguchi Y, Eid L, Parent M, et al. Exosome secretion is a key pathway for clearance of pathological TDP-43. Brain 2016;139:3187-201.

32 Münch C, O'Brien J, Bertolotti A. Prion-like propagation of mutant superoxide dismutase-1 misfolding in neuronal cells. Proc Natl Acad Sci U S A 2011; 108:3548-53.

33 Nicoll JA, Wilkinson D, Holmes C, et al. Neuropathology of human Alzheimer disease after immunization with amyloid-beta peptide: a case report. Nat Med 2003:9:448-52.

34 Masliah E, Hansen L, Adame A, et al. Abeta vaccination effects on plaque pathology in the absence of encephalitis in Alzheimer disease. Neurology 2005;64:129-31.

35 de Calignon A, Fox LM, Pitstick R, et al. Caspase activation precedes and leads to tangles. Nature 2010;464:1201-4.

36 Labbadia J, Morimoto RI. The biology of proteostasis in aging and disease. Annu Rev Biochem 2015:84:435-64

37 Nixon RA. The role of autophagy in neurodegenerative disease. Nat Med 2013:19:983-97.

38 Goldberg AL. Protein degradation and protection against misfolded or damaged proteins. Nature 2003:426:895-9.

39 Lokireddy S, Kukushkin NV, Goldberg AL. CAMP-induced phosphorylation of $26 \mathrm{~S}$ proteasomes on Rpn6/PSMD11 enhances their activity and the degradation of misfolded proteins. Proc Natl Acad Sci U SA 2015;112:E7176-E7185.

40 Myeku N, Clelland CL, Emrani S, et al. Tau-driven 26S proteasome impairment and cognitive dysfunction can be prevented early in disease by activating CAMP-PKA signaling. Nat Med 2016;22:46-53.

41 Watts GD, Wymer J, Kovach MJ, et al. Inclusion body myopathy associated with Paget disease of bone and frontotemporal dementia is caused by mutant valosincontaining protein. Nat Genet 2004;36:377-81.

42 Rubinsztein DC. The roles of intracellular protein-degradation pathways in neurodegeneration. Nature 2006;443:780-6.

43 Lowe J, Blanchard A, Morrell K, et al. Ubiquitin is a common factor in intermediate filament inclusion bodies of diverse type in man, including those of Parkinson's disease, Pick's disease, and Alzheimer's disease, as well as Rosenthal fibres in cerebellar astrocytomas, cytoplasmic bodies in muscle, and mallory bodies in alcoholic liver disease. J Pathol 1988;155:9-15.

44 Tofaris GK, Razzaq A, Ghetti B, et al. Ubiquitination of alpha-synuclein in Lewy bodies is a pathological event not associated with impairment of proteasome function.J Biol Chem 2003:278:44405-11.

45 Goode A, Butler K, Long J, et al. Defective recognition of LC3B by mutant SQSTM1/ p62 implicates impairment of autophagy as a pathogenic mechanism in ALS-FTLD. Autophagy 2016;12:1094-104

46 Chang D, Nalls MA, Hallgrímsdóttir IB, et al. A meta-analysis of genome-wide association studies identifies 17 new Parkinson's disease risk loci. Nat Genet 2017:49:1511-6.

47 Tofaris GK, Kim HT, Hourez R, et al. Ubiquitin ligase Nedd4 promotes alpha-synuclein degradation by the endosomal-lysosomal pathway. Proc Natl Acad Sci U S A 2011;108:17004-9.

48 Davies SE, Hallett PJ, Moens T, et al. Enhanced ubiquitin-dependent degradation by Nedd4 protects against $\alpha$-synuclein accumulation and toxicity in animal models of Parkinson's disease. Neurobiol Dis 2014;64:79-87.

49 Chung CY, Khurana V, Auluck PK, et al. Identification and rescue of $\alpha$-synuclein toxicity in Parkinson patient-derived neurons. Science 2013:342:983-7.

50 Alexopoulou Z, Lang J, Perrett RM, et al. Deubiquitinase Usp8 regulates $\alpha$-synuclein clearance and modifies its toxicity in Lewy body disease. Proc Natl Acad Sci U S A 2016:113:E4688-E4697.

51 Khurana V, Peng J, Chung CY, et al. Genome-scale networks link neurodegenerative disease genes to $\alpha$-Synuclein through specific molecular pathways. Cell Syst 2017:4:157-70.

52 Zhou Z, Kawabe H, Suzuki A, et al. NEDD4 controls spermatogonial stem cell homeostasis and stress response by regulating messenger ribonucleoprotein complexes. Nat Commun 2017;8:15662

53 Sun A, Wei J, Childress C, et al. The E3 ubiquitin ligase NEDD4 is an LC3-interactive protein and regulates autophagy. Autophagy 2017:13:522-37.

54 Lee BH, Lee MJ, Park S, et al. Enhancement of proteasome activity by a smallmolecule inhibitor of USP14. Nature 2010;467:179-84.

55 Lee JG, Takahama S, Zhang G, et al. Unconventional secretion of misfolded proteins promotes adaptation to proteasome dysfunction in mammalian cells. Nat Cell Biol 2016;18:765-76

56 Saigoh K, Wang YL, Suh JG, et al. Intragenic deletion in the gene encoding ubiquitin carboxy-terminal hydrolase in gad mice. Nat Genet 1999;23:47-51.

57 Papadopoulos C, Kirchner P, Bug M, et al. VCP/p97 cooperates with YOD1, UBXD1 and PLAA to drive clearance of ruptured lysosomes by autophagy. Embo J 2017:36:135-50.

58 Moreno JA, Radford H, Peretti D, et al. Sustained translational repression by elF2 $\alpha-P$ mediates prion neurodegeneration. Nature 2012;485:507-11 
59 Halliday M, Radford H, Zents KAM, et al. Repurposed drugs targeting elF2\&alpha;$\mathrm{P}$-mediated translational repression prevent neurodegeneration in mice. Brain 2017; 140:1768-83.

60 Perrett RM, Alexopoulou Z, Tofaris GK. The endosomal pathway in Parkinson's disease. Mol Cell Neurosci 2015;66:21-8.

61 Sidransky E, Nalls MA, Aasly JO, et al. Multicenter analysis of glucocerebrosidase mutations in Parkinson's disease. N Engl J Med 2009;361:1651-61.

62 Mazzulli JR, Xu YH, Sun Y, et al. Gaucher disease glucocerebrosidase and $\alpha$-synuclein form a bidirectional pathogenic loop in synucleinopathies. Cell 2011;146:37-52.

63 Aoki Y, Manzano R, Lee Y, et al. C9orf72 and RAB7L1 regulate vesicle trafficking in amyotrophic lateral sclerosis and frontotemporal dementia. Brain 2017;140:887-97.

64 Tanaka Y, Suzuki G, Matsuwaki T, et al. Progranulin regulates lysosomal function and biogenesis through acidification of lysosomes. Hum Mol Genet 2017;26:ddx011-88.

65 de la Monte SM, Luong T, Neely TR, et al. Mitochondrial DNA damage as a mechanism of cell loss in Alzheimer's disease. Lab Invest 2000;80:1323-35.

66 Swerdlow RH, Khan SM. A "mitochondrial cascade hypothesis" for sporadic Alzheimer's disease. Med Hypotheses 2004;63:8-20.

67 Panov AV, Gutekunst CA, Leavitt BR, et al. Early mitochondrial calcium defects in Huntington's disease are a direct effect of polyglutamines. Nat NeurosCi 2002;5:731-6

68 Du H, Guo L, Yan S, et al. Early deficits in synaptic mitochondria in an Alzheimer's disease mouse model. Proc Natl Acad Sci U S A 2010;107:18670-5.

69 Wang L, Guo L, Lu L, et al. Synaptosomal mitochondrial dysfunction in 5xFAD mouse model of Alzheimer's disease. PLoS One 2016:11:e0150441.

70 Di Maio R, Barrett PJ, Hoffman EK, et al. $\alpha$-Synuclein binds to TOM20 and inhibits mitochondrial protein import in Parkinson's disease. Sci Trans/ Med 2016;8:ra78ra78.

71 Pera M, Larrea D, Guardia-Laguarta C, et al. Increased localization of APP-C99 in mitochondria-associated ER membranes causes mitochondrial dysfunction in Alzheimer disease. Embo J 2017;36:3356-71.

72 Wang W, Wang L, Lu J, et al. The inhibition of TDP-43 mitochondrial localization blocks its neuronal toxicity. Nat Med 2016;22:869-78.

73 Lopez-Gonzalez R, Lu Y, Gendron TF, et al. Poly(GR) in C90RF72-related ALS/ FTD compromises mitochondrial function and increases oxidative stress and DNA damage in iPSC-derived motor neurons. Neuron 2016;92:383-91.

74 Sugars KL, Rubinsztein DC. Transcriptional abnormalities in Huntington disease. Trends Genet 2003;19:233-8.

75 McWilliams TG, Muqit MM. PINK1 and Parkin: emerging themes in mitochondrial homeostasis. Curr Opin Cell Biol 2017;45:83-91.

76 Du F, Yu Q, Yan S, et al. PINK1 signalling rescues amyloid pathology and mitochondrial dysfunction in Alzheimer's disease. Brain 2017;140:3233-51.

77 McWilliams TG, Prescott AR, Montava-Garriga L, et al. Basal mitophagy occurs independently of PINK1 in mouse tissues of high metabolic demand. Cell Metab 2018.

78 Lambert JC, Ibrahim-Verbaas CA, Harold D, et al. Meta-analysis of 74,046 individuals identifies 11 new susceptibility loci for Alzheimer's disease. Nat Genet 2013:45:1452-8.

79 Guerreiro R, Wojtas A, Bras J, et al. TREM2 variants in Alzheimer's disease. N Engl J Med 2013:368:117-27.

80 Jonsson T, Stefansson $H$, Steinberg $S$, et al. Variant of TREM2 associated with the risk of Alzheimer's disease. N Engl J Med 2013;368:107-16.

81 Rayaprolu S, Mullen B, Baker M, et al. TREM2 in neurodegeneration: evidence for association of the p.R47H variant with frontotemporal dementia and Parkinson's disease. Mol Neurodegener 2013;8:19.

82 Colonna M. TREMs in the immune system and beyond. Nat Rev Immunol 2003:3:445-53.

83 Tahara K, Kim HD, Jin JJ, et al. Role of toll-like receptor signalling in Abeta uptake and clearance. Brain 2006;129:3006-19.

$84 \mathrm{Kim} \mathrm{C}, \mathrm{Ho} \mathrm{DH}$, Suk JE, et al. Neuron-released oligomeric $\alpha$-synuclein is an endogenous agonist of TLR2 for paracrine activation of microglia. Nat Commun 2013:4:1562.

85 Wang Y, Ulland TK, Ulrich JD, et al. TREM2-mediated early microglial response limits diffusion and toxicity of amyloid plaques. J Exp Med 2016;213:667-75.

86 Yuan P, Condello C, Keene CD, et al. TREM2 haplodeficiency in mice and humans impairs the microglia barrier function leading to decreased amyloid compaction and severe axonal dystrophy. Neuron 2016;92:252-64.

87 Leyns CEG, Ulrich JD, Finn MB, et al. TREM2 deficiency attenuates neuroinflammation and protects against neurodegeneration in a mouse model of tauopathy. Proc Natl Acad Sci U S A 2017:114:11524-9.

88 Hong S, Stevens B. Microglia: phagocytosing to clear, sculpt, and eliminate. Dev Cell 2016:38:126-8.

89 Hong S, Beja-Glasser VF, Nfonoyim BM, et al. Complement and microglia mediate early synapse loss in Alzheimer mouse models. Science 2016;352:712-6.

90 Stevens B, Allen NJ, Vazquez LE, et al. The classical complement cascade mediates CNS synapse elimination. Cell 2007;131:1164-78.

91 Lui H, Zhang J, Makinson SR, et al. Progranulin deficiency promotes circuit-specific synaptic pruning by microglia via complement activation. Cell 2016;165:921-35.
92 Gitcho MA, Baloh RH, Chakraverty S, et al. TDP-43 A315T mutation in familial motor neuron disease. Ann Neurol 2008:63:535-8.

93 Kabashi E, Valdmanis PN, Dion P, et al. TARDBP mutations in individuals with sporadic and familial amyotrophic lateral sclerosis. Nat Genet 2008;40:572-4

94 Sreedharan J, Blair IP, Tripathi VB, et al. TDP-43 mutations in familial and sporadic amyotrophic lateral sclerosis. Science 2008;319:1668-72.

95 Kwiatkowski TJ, Bosco DA, Leclerc AL, et al. Mutations in the FUS/TLS gene on chromosome 16 cause familial amyotrophic lateral sclerosis. Science 2009;323:1205-8.

96 Vance C, Rogelj B, Hortobágyi T, et al. Mutations in FUS, an RNA processing protein, cause familial amyotrophic lateral sclerosis type 6. Science 2009;323:1208-11.

97 Van Deerlin VM, Leverenz JB, Bekris LM, et al. TARDBP mutations in amyotrophic lateral sclerosis with TDP-43 neuropathology: a genetic and histopathological analysis. Lancet Neurol 2008;7:409-16.

98 Buratti E, Dörk T, Zuccato E, et al. Nuclear factor TDP-43 and SR proteins promote in vitro and in vivo CFTR exon 9 skipping. Embo J 2001;20:1774-84.

99 Ling JP, Pletnikova O, Troncoso JC, et al. TDP-43 repression of nonconserved cryptic exons is compromised in ALS-FTD. Science 2015;349:650-5.

100 Shiga A, Ishihara T, Miyashita A, et al. Alteration of POLDIP3 splicing associated with loss of function of TDP-43 in tissues affected with ALS. PLoS One 2012;7:e43120.

101 Tollervey JR, Curk T, Rogelj B, et al. Characterizing the RNA targets and positiondependent splicing regulation by TDP-43. Nat Neurosci 2011;14:452-8.

102 Liu X, Li D, Zhang W, et al. Long non-coding RNA gadd7 interacts with TDP-43 and regulates Cdk6 mRNA decay. Embo J 2012;31:4415-27.

103 Costessi L, Porro F, laconcig A, et al. TDP-43 regulates $\beta$-adducin (Add2) transcript stability. RNA Biol 2014;11:1280-90.

104 Murakami T, Qamar S, Lin JQ, et al. ALS/FTD Mutation-induced phase transition of FUS liquid droplets and reversible hydrogels into irreversible hydrogels impairs RNP granule function. Neuron 2015;88:678-90.

105 Chou CC, Zhang Y, Umoh ME, et al. TDP-43 pathology disrupts nuclear pore complexes and nucleocytoplasmic transport in ALS/FTD. Nat Neurosci 2018

106 Freibaum BD, Lu Y, Lopez-Gonzalez R, et al. GGGGCC repeat expansion in C9orf72 compromises nucleocytoplasmic transport. Nature 2015;525:129-33.

107 Polak U, Mclvor E, Dent SY, et al. Expanded complexity of unstable repeat diseases. Biofactors 2013:39:164-75.

108 Li LB, Yu Z, Teng X, et al. RNA toxicity is a component of ataxin-3 degeneration in Drosophila. Nature 2008;453:1107-11.

109 Mutsuddi M, Marshall CM, Benzow KA, et al. The spinocerebellar ataxia 8 noncoding RNA causes neurodegeneration and associates with staufen in Drosophila. Curr Biol 2004; 14:302-8

110 Koob MD, Moseley ML, Schut LJ, et al. An untranslated CTG expansion causes a novel form of spinocerebellar ataxia (SCA8). Nat Genet 1999;21:379-84.

111 Moseley ML, Zu T, Ikeda Y, et al. Bidirectional expression of CUG and CAG expansion transcripts and intranuclear polyglutamine inclusions in spinocerebellar ataxia type 8. Nat Genet 2006;38:758-69.

112 Wilburn B, Rudnicki DD, Zhao J, et al. An antisense CAG repeat transcript at JPH3 locus mediates expanded polyglutamine protein toxicity in Huntington's disease-like 2 mice. Neuron 2011:70:427-40.

113 Hollingsworth EW, Vaughn JE, Orack JC, et al. iPhemap: an atlas of phenotype to genotype relationships of human iPSC models of neurological diseases. EMBO Mol Med 2017;9:1742-62

114 Israel MA, Yuan SH, Bardy C, et al. Probing sporadic and familial Alzheimer's disease using induced pluripotent stem cells. Nature 2012;482:216-20.

115. Dafinca R, Scaber J, Ababneh N, et al. C9orf72 Hexanucleotide. expansions are associated with altered endoplasmic reticulum calcium homeostasis and stress granule formation in induced pluripotent stem cell-derived neurons from patients with amyotrophic lateral sclerosis and frontotemporal dementiaStem Cells 2016;34:2063-78

116 Silva MC, Cheng C, Mair W, et al. Human iPSC-derived neuronal model of TauA152T frontotemporal dementia reveals tau-mediated mechanisms of neuronal vulnerability. Stem Cell Reports 2016;7:325-40.

117 Fernandes HJ, Hartfield EM, Christian HC, et al. ER Stress and autophagic perturbations lead to elevated extracellular $\alpha$-synuclein in GBA-N370S Parkinson's iPSC-derived dopamine neurons. Stem Cell Reports 2016:6:342-56.

118 Heman-Ackah SM, Manzano R, Hoozemans JJM, et al. Alpha-synuclein induces the unfolded protein response in Parkinson's disease SNCA triplication iPSC-derived neurons. Hum Mol Genet 2017;26:4441-50.

119 Sandoe J, Eggan K. Opportunities and challenges of pluripotent stem cell neurodegenerative disease models. Nat Neurosci 2013;16:780-9.

120 Jinek M, Chylinski K, Fonfara I, et al. A programmable dual-RNA-guided DNA endonuclease in adaptive bacterial immunity. Science 2012;337:816-21.

121 Doudna JA, Charpentier E. Genome editing. The new frontier of genome engineering with CRISPR-Cas9. Science 2014;346:1258096.

122 Thakore PI, Black JB, Hilton IB, et al. Editing the epigenome: technologies for programmable transcription and epigenetic modulation. Nat Methods 2016;13:127-37. 